\title{
Hybrid wireless communication link
}

J. Mikołajczyk, R. Matyszkiel, D. Szabra, A. Prokopiuk, B. Grochowina, et al.

J. Mikołajczyk, R. Matyszkiel, D. Szabra, A. Prokopiuk, B. Grochowina, Z. Bielecki, "Hybrid wireless communication link," Proc. SPIE 11442,

Radioelectronic Systems Conference 2019, 114420K (11 February 2020); doi: $10.1117 / 12.2565289$

SPIE. Event: Radioelectronic Systems Conference 2019, 2019, Jachranka, Poland 


\title{
Hybrid wireless communication link
}

\author{
J.Mikolajczyk*a, R.Matyszkiel $^{\text {b }}$, D.Szabra ${ }^{\text {a }}$, A.Prokopiuk ${ }^{\text {a }}$, B.Grochowina ${ }^{\text {b }}$, Z.Bielecki ${ }^{\text {a }}$ \\ anstitute of Optoelectronics MUT, 2 Kaliskiego St., 00-908 Warsaw, Poland \\ bMilitary Communication Institute, 22A Warszawska St., 05-130 Zegrze, Poland
}

\begin{abstract}
The paper presents a construction of a wireless hybrid data link operates applying two transmission channels using optical radiation (FSO - Free Space Optics) and radio one (RF - Radio Frequency). Based on some parameters (e.g. laser power, optics aperture, photodetector detectivity, signal bandwidth, beam divergence) of the link components, its data range was determined for various operating conditions (visibility and turbulence). Preliminary tests of the link prototype (TRL 6) were carried out at the Military Communication Institute, Poland. The results showed that the use of FSO/RF technology systems can provide the increase in data transmission security, link availability, and immunity to intentional interference. Considering the features of this technology, high applicable potential to military activities was observed.
\end{abstract}

Keywords: wireless communications, quantum cascade lasers, free space optics, data transmission security, hybrid data link

\section{INTRODUCTION}

Nowadays, military wireless communication systems work using radio technology, which provides a high availability data link over a large area in different weather conditions. However, there are several limiting factors of using radiocommunication systems: spectrum license [1], influence of modern electronic warfare systems [2], electromagnetic compatibility of the system components, especially those operating within one object (ensuring co-site compatibility) [3]. The application of co-site radio communication devices increases requirements for a radio planning system. The main task of the radio planning system is to generate and distribute radio data considering parameters and interference criteria. These criteria enable the action of the whole radio system (ensuring internal compatibility of the system components). In some situations, limited spectral resources make it impossible to ensure optimal operation of the communication system (due to power reduction of the useful signal in the given radio relations). Therefore, there is a permanent necessity to develop alternative wireless communication technology without any co-site separation and spectral license requirements. The application of radio/optical hybrid data link makes it possible to overcome such capability gaps.

The designed hybrid link consists of two data transmission channels using optical radiation (FSO) and radio waves (RF). Unlike existing FSO/RF communication devices, the applied optical channel operates in the spectral range of 8-12 $\mu \mathrm{m}$ (LWIR) applying quantum cascade lasers and MCT photodetectors [4]. That is why, it is less sensitive to worse atmospheric phenomena (fog, mist or turbulence) and harder to detect, interception of data transmissions comparing currently used FSO systems operated in the near infrared (900 nm - NIR) or short wave infrared (1500 nm - SWIR) range and lower risk of interferences [5]. Because radio links are characterized by low signal attenuation in bad weather conditions (e.g. fog and turbulence), the integration of both technologies into one FSO/RF device allows increasing data link availability up to the value of $99.999 \%$ [6,7]. This is very important for military operations [8]:

- increasing the security of data transmission;

- increasing the level of link availability;

- reducing the probability of detection;

- increasing resistance to intentional interference.

A simplified hybrid data link diagram is shown in Fig.1.

*janusz.mikolajczyk @wat.edu.pl, phone 48261 839 792; zdso.wat.edu.pl

Radioelectronic Systems Conference 2019, edited by Piotr Kaniewski, Jan Matuszewski, Proc. of SPIE Vol. 11442, 114420K · C 2020 SPIE · CCC code: 0277-786X/20/\$21 · doi: 10.1117/12.2565289 


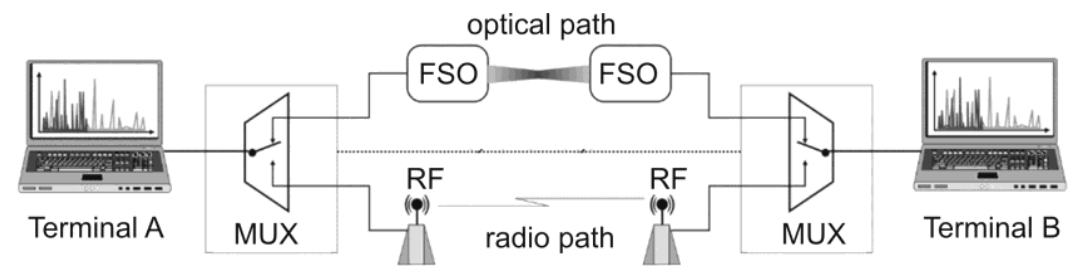

Figure 1. Schematic diagram of the FSO/RF hybrid data link, FSO - optical channel, RF - radio channel, MUX multiplexing system.

\section{FSO/RF HYBRID LINK PROTOTYPE}

Analysis of the FSO/RF device construction provides to define key technologies influencing its parameters and functionality. The schematic diagram of FSO/RF data link is shown in Fig. 2. The cooperation algorithm of FSO and RF transmission channels has been implemented with the analysis of the quality data transmission in both channels.

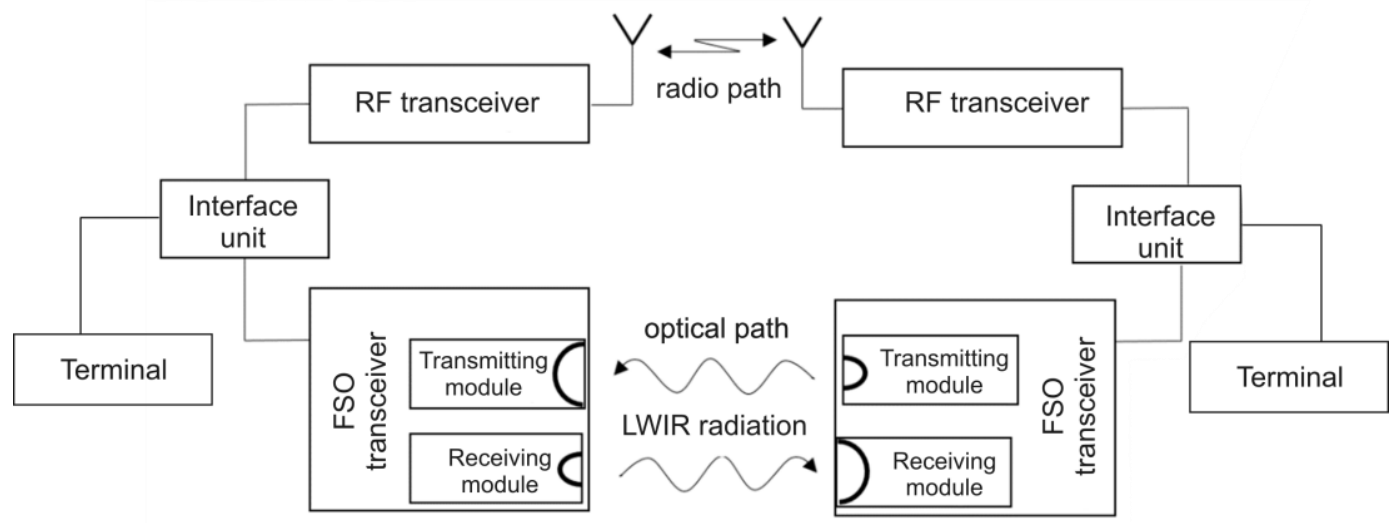

Figure 2. Block diagram of the FSO/RF hybrid link prototype.

In the FSO transmitter, quantum cascade lasers emitting radiation at the wavelength of $9.3 \mu \mathrm{m}$ were used. An active power regulation of the transmitted radiation is performed using a unique optoelectronic system. Implemented algorithm provides to not only stabilize laser pulse power and reaching the receiver but also to identify of beam interruption (accidental or intentional ones). It is important because of potential actions related to data transmission failure. This can be happened by:

- disturbances of the field of view of the transmitting and receiving systems caused by atmospheric conditions or external objects,

- malfunction of the transmitting and/or receiving path components.

The mechanical construction integrates whole components of the FSO transceiver (Fig. 3).
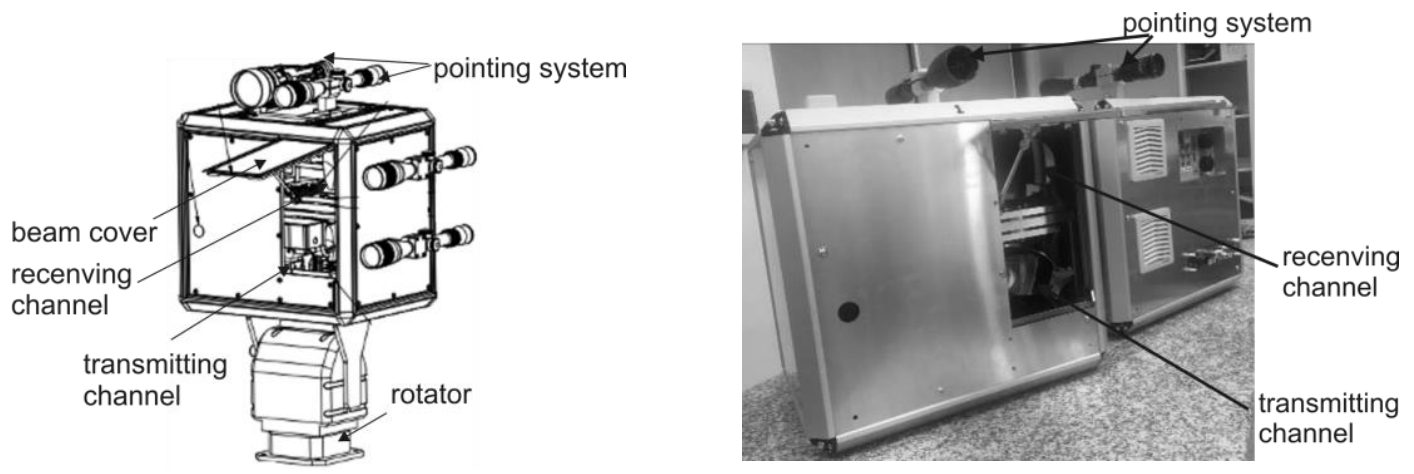

Figure 3. 3D model and view of the FSO head (transceiver and receiver). 


\section{PRELIMINARY TESTS}

The main task of preliminary tests was to examine the FSO/RF prototype functionalities, i.e. the availability of the data link, transmission rate and BER (Bit Error Rate) of both FSO and RF channels, and time of remote channels reconfigurations. Figure 4 presents measurement conditions and view of the FSO transceiver/receiver equipped with a GPS positioning system. As a radio channel, WLAN was used.
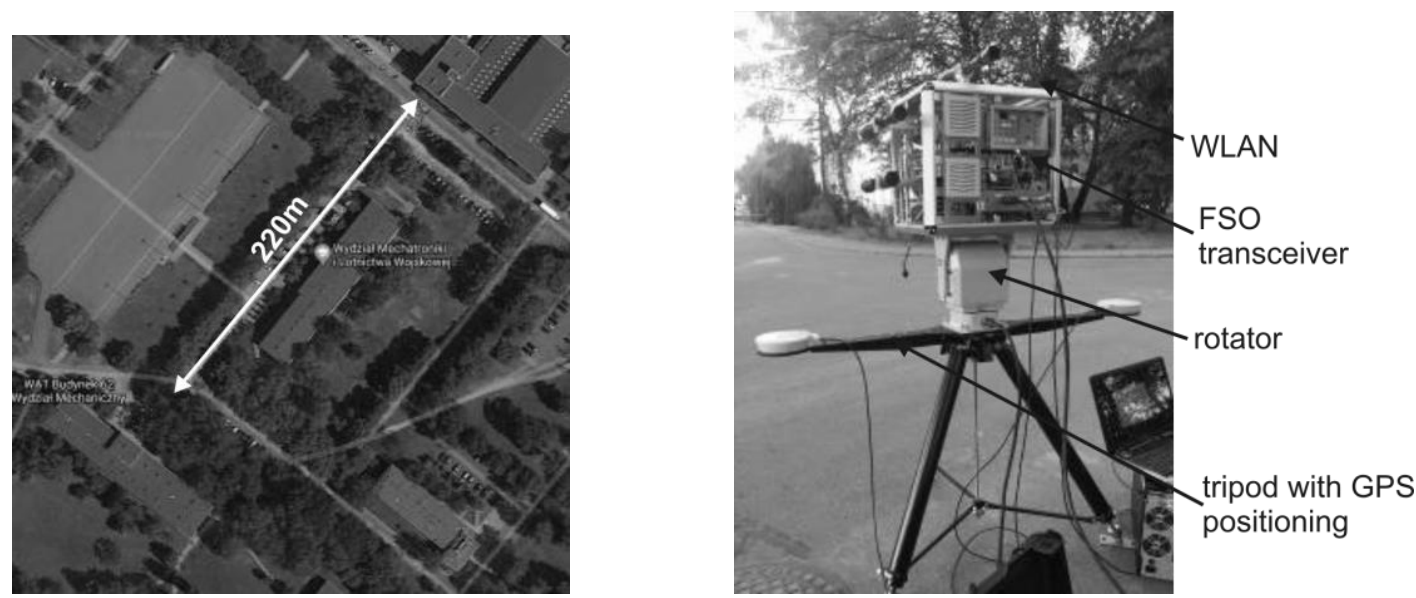

Figure 4. View of the test conditions and the FSO transceiver with GPS positioning system.

Tests of the hybrid link availability were performed using a transceiver shutter to simulate laser beam disruption or disconnecting the WLAN antenna. During this procedure, the switching times of each channel were measured. In the next step, the transmission rate, packet losses and the delay time were measured using the Netperf software. To determine the bit error rate, a special HYBRYDOL ${ }^{\circledR}$ software (KenBIT Koenig i Wspólnicy Sp.j.) was used. This software compares the pseudo-random data streams transmitted in the packet in a coded and an un-coded form with the corresponding signals generated locally. The ratio of the number of error bits to the total received bits corresponds to the BER value. The results of preliminary tests are summarized in Table 1.

Table 1 . The results of preliminary tests.

\begin{tabular}{|c|l|l|}
\hline \multicolumn{2}{|c|}{ Test name } & \multicolumn{2}{|c|}{ Result } \\
\hline \multirow{2}{*}{ Automatic switching time } & FSO $\rightarrow \mathrm{RF}$ & $28 \mathrm{~s}$ \\
\cline { 2 - 3 } & $\mathrm{RF} \rightarrow \mathrm{FSO}$ & $18 \mathrm{~s}$ \\
\hline \multirow{2}{*}{ Control switching time } & FSO $\rightarrow \mathrm{RF}$ & $23 \mathrm{~s}$ \\
\cline { 2 - 3 } & $\mathrm{RF} \rightarrow \mathrm{FSO}$ & $12 \mathrm{~s}$ \\
\hline \multirow{2}{*}{ Transmission rate } & FSO & $10.9 \mathrm{Mb} / \mathrm{s}$ \\
\cline { 2 - 3 } & $\mathrm{RF}$ & $37 \mathrm{Mb} / \mathrm{s}$ \\
\hline \multirow{2}{*}{$\begin{array}{c}\text { BER level for different defined transmitter } \\
\text { radiation power levels }\end{array}$} & very low $[20 \%]$ & no link \\
\cline { 2 - 3 } & low $[40 \%]$ & $10^{-2}$ \\
\cline { 2 - 3 } & medium $[60 \%]$ & $10^{-5}$ \\
\cline { 2 - 3 } & high $[80 \%]$ & 0 \\
\cline { 2 - 3 } & very high $[100 \%]$ & 0 \\
\hline
\end{tabular}

\section{RANGE ANALYSIS OF OPTICAL LINK TRANSMISSION}

The data transmission range is mainly defined by some optical path properties. The analysis of the beam light attenuation should mainly consider absorption, scattering, and turbulence phenomena. The first one was minimized working in socalled atmosphere transmission window. To determine level of radiation scattering, extinction coefficient value $(\gamma)$ is analyzed. Its value is determined using simulation software (PcMODWIN) or analytical models, e.g. basing on visibility. 
For example, extinction coefficient values for different visibilities and two wavelengths of $1.5 \mu \mathrm{m}$ and $10 \mu \mathrm{m}$ basing on the Kim model were determined (Fig. 5).

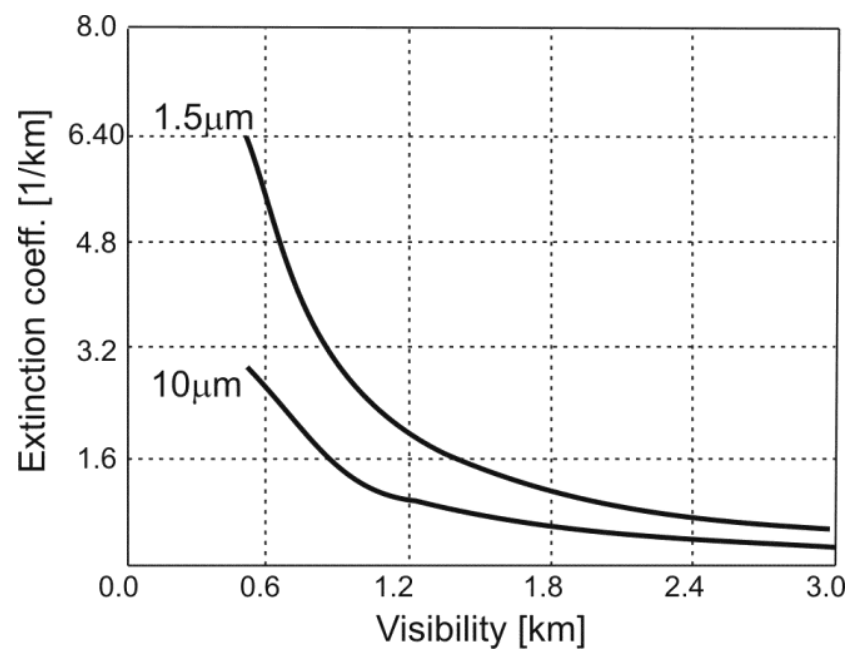

Figure 5. Extinction coefficient vs. visibility for two wavelengths: $10 \mu \mathrm{m}$ and $1.5 \mu \mathrm{m}$.

For visibility values, appropriate atmospheric conditions can be defined. For example, the visibility range of $50 \mathrm{~m} \div 200 \mathrm{~m}$ corresponds to dense fog or very dense snow, while the $2 \mathrm{~km} \div 4 \mathrm{~km}$ range is equivalent to the appearance of fog, heavy rain or moderate snow. For these visibility ranges, the extinction coefficient is in the range of $78.2 \div 19.6$ and $1.960 \div 0.954$, respectively [9]. Weather conditions have strong impact on the radiation attenuation and can limit the FSO data range. However, operation in the longer wavelength range (LWIR) can provide an increase in the link range compared to the shorter ones, i.e. NIR and SWIR (Fig. 6).

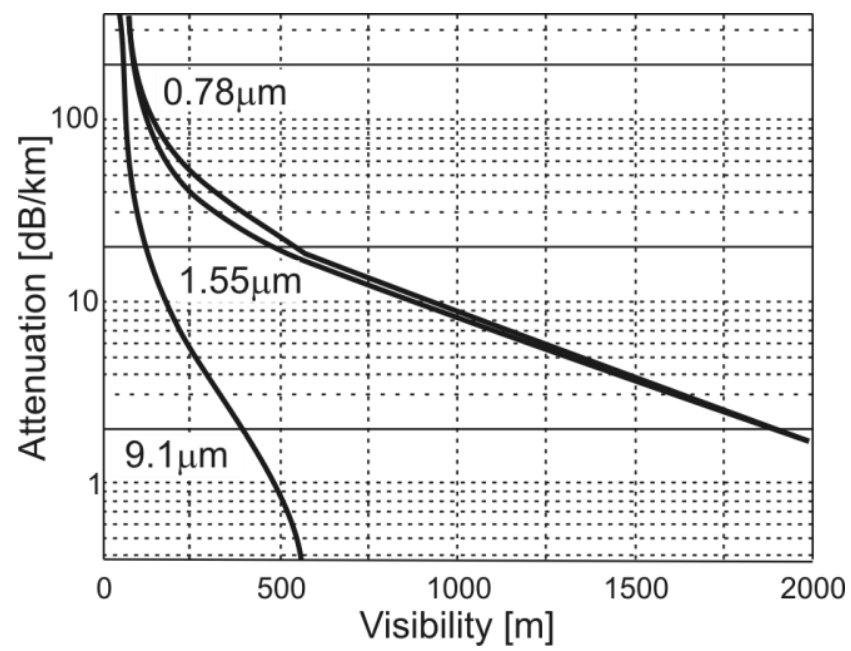

Figure 6. Dependence of visibility on attenuation coefficient for three spectral ranges: NIR, SWIR and LWIR.

Turbulence is an important factor that can significantly attenuate registered optical pulses. It is characterized by the refractivity turbulence structure constant $C_{\mathrm{n}}^{2}$. This constant takes values from $10^{-17} \mathrm{~m}^{-2 / 3}$ (weak turbulence) to $10^{-12} \mathrm{~m}^{-2 / 3}$ (very strong turbulence). The $\mathrm{C}_{\mathrm{n}}{ }^{2}$ parameter depends on many factors and varies with the height of the FSO device location, type of surface over which radiation is transmitted, temperature and pressure distribution, and even the wind speed. There are many analytical models to determine the value of this parameter depending on climate features. $\mathrm{C}_{\mathrm{n}}{ }^{2}$ dependence on the FSO height determined using the Gurvich model is shown in Fig. 7 [10]. 


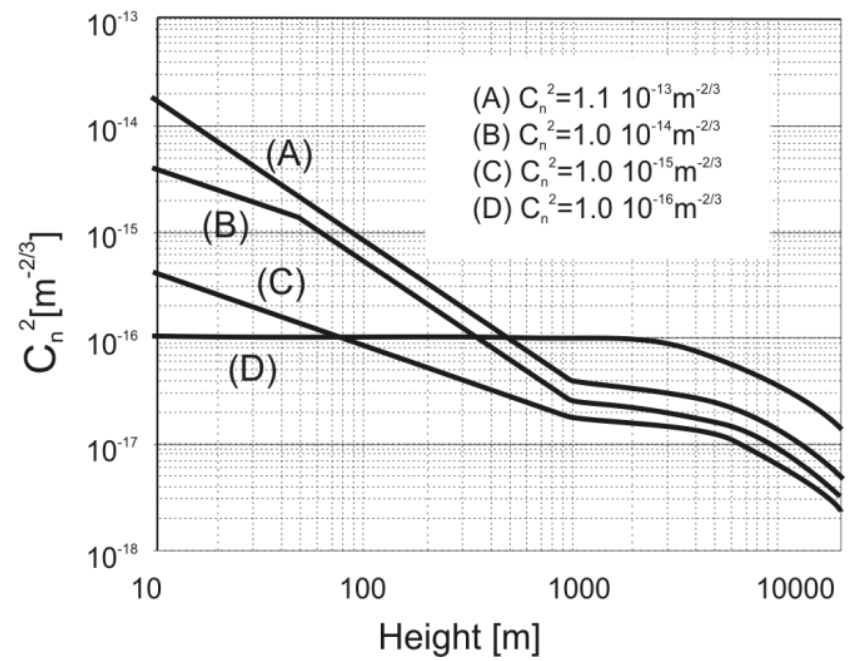

Figure 7. $\mathrm{C}_{\mathrm{n}}^{2}$ parameter vs. height determined for the Gurvich model with four turbulence levels [10]

The analytical analysis of the data range was performed basing on parameters of the final construction of the FSO prototype. The SNR values for some different turbulence levels (values of the $\mathrm{C}_{\mathrm{n}}^{2}$ parameter) and weather conditions (the extinction coefficient $\gamma$ ) were determined (Fig. 8).
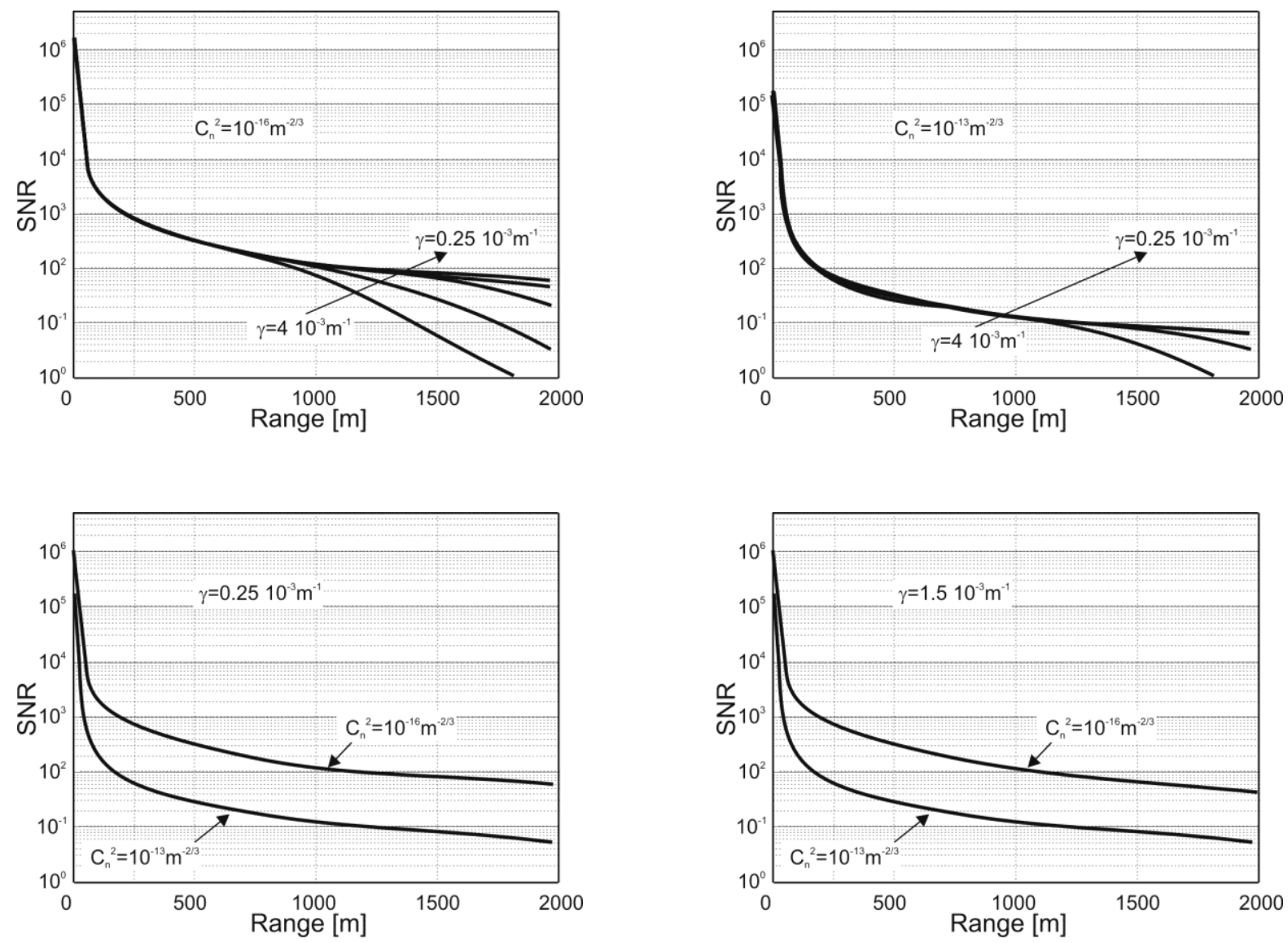

Figure 8. SNR dependence on the data link range of the developed FSO prototype for various weather conditions (determined by $\gamma=0.25 \mathrm{~m}^{-1}$ - visibility better than $10 \mathrm{~km}$, and by $\gamma=4 \mathrm{~m}^{-1}$, visibility of $0.5 \mathrm{~km}$ ) 
The effect of turbulence is especially evident in the short distances. As the range growths, the effect of scattering is also increasing. Strong turbulence can significantly reduce data transfer. It will take place if the laser beam propagates near the earth's surface, as well as in the presence of high dynamics of temperature or wind speed changes. However, the application of an extra RF data link in hybrid configuration provides data transfer over a given distance.

\section{CONCLUSION}

The preliminary results of FSO/RF hybrid data link show $n$ its potential to ensure point-to-point communications between command posts, stationary or mobile infrastructure at various levels of command (division, brigade, battalion) using mobile communication nodes. The use of FSO/RF technology makes it possible to replace the previously used radio devices in some scenarios in order to reduce the demand for spectrum and improves the compatibility of the work of other radio devices in the system. In addition, the use of FSO/RF hybrid devices makes wireless communications more resistant to the impact of electronic warfare systems with the increased availability of $99.999 \%$.

\section{ACKNOWLEDGMENTS}

This research was supported by The Polish National Centre for Research and Development grant DOBBIO8/01/01/2016.

\section{REFERENCES}

[1] Suchański, M., Kaniewski, P., Matyszkiel, R. and Gajewski, P., "Dynamic spectrum management in legacy military communication systems," 2012 Mil. Commun. Inf. Syst. Conf., 1-5 (2012).

[2] Matyszkiel, R., Polak, R., Lubkowski, P. and Laskowski, D., "Mechanisms of immunization of broadband radio stations for targeted interference," XII Conf. Reconnaiss. Electron. Warf. Syst. 11055, 110550G (2019).

[3] Kosmowski, K. and Matyszkiel, R., "Verification of the criterion and measures of interferences used in radio planning systems," XII Conf. Reconnaiss. Electron. Warf. Syst. 11055, 110550J (2019).

[4] Mikołajczyk, J., Bielecki, Z., Bugajski, M., Piotrowski, J., Wojtas, J., Gawron, W., Szabra, D. and Prokopiuk, A., "Analysis of Free-Space Optics Development," Metrol. Meas. Syst., 653-674 (2017).

[5] Leitgeb, E., Plank, T., Awan, M. S., Brandl, P., Popoola, W., Ghassemlooy, Z., Ozek, F. and Wittig, M., "Analysis and evaluation of optimum wavelengths for free-space optical transceivers," 2010 12th Int. Conf. Transparent Opt. Networks, 1-7 (2010).

[6] Matyszkiel, R., Polak, R., Kaniewski, P. and Laskowski, D., "The results of transmission tests of polish broadband SDR radios," 2017 Commun. Inf. Technol., 1-6 (2017).

[7] Wisniewski, M., Dobkowski, A., Pater, G., Matyszkiel, R., Kaniewski, P. and Grochowina, B., "Test results of polish SDR narrowband radio," 2017 Commun. Inf. Technol., 1-6 (2017).

[8] Mikołajczyk, J., Szabra, D., Matyszkiel, R. and Grochowina, B., "Possibilities of Using FSO/RF Technology in Military Communication Systems," 2018 New Trends Signal Process., 1-4 (2018).

[9] Guo, F., Tang, J. and Xiao, X., "Foggy scene rendering based on transmission map estimation," Int. J. Comput. Games Technol. 2014, 1-13 (2014).

[10] Ghassemlooy, Z., Popoola, W. and Rajbhandari, S., [Optical wireless communications: system and channel modelling with Matlab $\left.{ }^{\circledR}\right]$, CRC press (2019). 